\title{
Hyaluronic Acid Mediated Enrichment of CD44 Expressing Glioblastoma Stem Cells in U251MG Xenograft Mouse Model
}

Arun Vaidyanath", Hafizah Binti Mahmud", Apriliana Cahya Khayrani, Aung KoKo Oo, Akimasa Seno, Mami Asakura, Tomonari Kasai* and Masaharu Seno

Department of Medical Bioengineering Science, Okayama University, Japan

"These authors have contributed equally to the article

\begin{abstract}
Background: Glioblastoma is one of the most aggressive cancer with high mortality rates and poses several hurdles in the efficient chemotherapeutic intervention. Similar to other cancers, glioma also harbors CSCs, that are self-renewable, multipotent cells, which initiate the cancer incidence, chemotherapeutic resistance and cancer recurrence. The microenvironmental regulation in the brain tumor and metastasis involves the cooperative interaction between HA and CD44. CD44, being a multifaceted transmembrane glycoprotein by itself, or in combination with several other cell surface receptors, has been used as a marker for CSC isolation.
\end{abstract}

Methods: We established both adherent and nonadherent culture of U251MG cells by treating with high molecular weight HA. Further these cells were transplanted subcutaneously in Balb/c mouse for the generation of the xenograft model for the cancer stem cell. The tumor was further characterized for the establishment of the working model for molecular targeting studies of cancer stem cells.

Results: Here we showed the enrichment of the CD44 expressing population of glioblastoma cells by induction with hyaluronic acid. The non-adherent culture spheroids of U251MG cells showed up regulation in the CD44 expression along with aberrant activation of principal pluripotency genes OCT3/4, SOX2, KLF4 and Nanog. Using the HA-treated spheroid, we established an experimental xenograft mouse model with high angiogenesis enhanced tumor-initiating capacity while retaining the glioblastoma traits.

Conclusion: We characterized a mouse xenograft model of U251MG cells which could be a promising model system to study the molecular targeting approaches against CSCs in glioblastoma.

Keywords: HA; CD44; Cancer stem cell; Xenograft; Mouse model

\section{Introduction}

Glioblastoma multiforme is the one of the most malignant cancer affecting the brain making them one of the incurable cancers with a survival rate of $5 \%$. Several studies have shed light on the cytogenetic, molecular and epigenetic alterations during its incidence and have characterized the tumor to its molecular level [1]. Histopathological attributes have helped in the classifying gliomas into two broad lineages: astrocytoma and oligodendroma and four grades (I-IV) of which the grade I and II corresponds to the low-grade glioma and III and IV correspond to the high-grade gliomas [2]. Glioblastoma cells are noninvasive outside the brain partly because of the effective blood brain barrier and the multitude of the complex microenvironment which it requires for the metastasis [3]. With the progression of the tumor metastatic cells uses the neovascularization for the spread of cancer and the blood-brain barrier changes to a blood-tumor barrier. Considering the microenvironment in the brain and its relative complexity in targeting the blood-tumor barrier are less receptive to chemotherapeutic intervention [4]. Glioma, like other cancers, are highly heterogeneous and shows hierarchy in their progression with the apex being the Cancer Stem Cell (CSCs) which repopulates the whole of the tumor. The CSCs are believed to be the primordial reason for the recurrence and reinstatement of the tumor after chemotherapeutic intervention and surgery as they are radiation and chemotherapeutic resistant [5]. The perpetual selfrenewability of the CSC is a complex process, which is mediated by the cellular and soluble attributes of the tumor microenvironment which includes the extracellular matrix, mesenchymal cells, endothelial cells and secretory molecules comprising cytokines and growth factor. The microenvironments surrounding the CSCs are termed as niche which modulates the cellular fate of the CSC [5-7].
CD44 is a multifunctional transmembrane glycoprotein which is a commonly used marker for isolating the CSCs from various cancers and mediating cell-cell and cell-matrix interactions furthering the malignancy and cancer dissemination. CD44 is typically associated with CD24, CD133, and CD34 for the enrichment of the various cancers. Hyaluronic Acid (HA) biosynthesis in the tumor microenvironment has been shown to increase the cancer aggressiveness and poor clinical outcome affecting the overall survival rate of the patients [8,9]. Our previous study with our platform of microarray method showed that the CD44 is overexpressed in several of the glioma cancer cells analyzed [10]. In breast cancer cells, HA overexpression drives EMT which is one of the key aspects of the CSC generation and propagation [11]. Over recent years CD44 along with other cell surface markers like CD133, CD90, CD24, ALDH, Nestin, EpCAM, and CD34 are also associated with CSC isolation and enrichment in various tumors [12]. In addition to its naturally occurring ligand, CD44 interacts with several ECM molecules including fibronectin galectin-8, laminin, fibrinogen, chondroitin sulfate [13], and osteopontin [14].

*Corresponding authors: Tomonari Kasai, Laboratory of Nano-Biotechnology, Department of Medical Bioengineering Science, Faculty of Engineering, Graduate School of Natural Science and Biotechnology, Okayama University, 3.1.1 Tsushima-Naka, Kita-ku, Okayama 700-8530, Japan, Tel: +81-86-2518265; E-mail: t-kasai@okayama-u.ac.jp

Received March 20, 2017; Accepted April 03, 2017; Published April 10, 2017

Citation: Vaidyanath A, Mahmud HB, Khayrani AC, Oo AKK, Seno A, et al. (2017) Hyaluronic Acid Mediated Enrichment of CD44 Expressing Glioblastoma Stem Cells in U251MG Xenograft Mouse Model. J Stem Cell Res Ther 7: 384. doi: 10.4172/2157-7633.1000384

Copyright: (C) 2017 Vaidyanath A, et al. This is an open-access article distributed under the terms of the Creative Commons Attribution License, which permits unrestricted use, distribution, and reproduction in any medium, provided the original author and source are credited. 
Targeted delivery of the chemotherapeutic compounds to the CSCs will be beneficial for the regression and recurrence of cancer. In this report, we are analyzing the enrichment of CD44 expression in spheroid culture with the addition of HA. Utilizing the spheroid culture, we developed a mouse glioblastoma tumor model for targeted drug delivery approaches for CSCs.

\section{Materials and Methods}

\section{Cell culture}

U251MG, SK-OV-3, and A172 were cultured as per the protocol described elsewhere [10]. For primary cultures mouse xenografts were cut into small pieces of $1 \mathrm{~mm}^{3}$ in HBSS. The tissues were further washed three times and were transferred to a $15-\mathrm{ml}$ conical tube with $0.25 \%$ trypsin (3-4 fold) for $30 \mathrm{~min}$ at $37^{\circ} \mathrm{C}$ and were stopped by the addition of media containing FBS. The cell suspension was spun down at $800 \mathrm{rpm}$ for $10 \mathrm{~min}$. The cell pellet was washed with HBSS and was suspended in complete media and seeded into a dish at a density of $5-6 \times 10^{5} / \mathrm{ml}$. Cells were passaged every 3 days and cells morphology was observed and photographed using Olympus IX81 (Olympus, Japan) microscope equipped with a light fluorescence device

\section{Sphere formation and HA treatment}

For the sphere formation assay, the cell number were adjusted to 1 $\times 10^{4} / \mathrm{ml}$ and were cultured in ultra-low attachment dishes in the media devoid of FBS and containing Insulin/Transferrin/Selenium-X (ITS-X) and $100 \mu \mathrm{g} / \mathrm{ml} \mathrm{HA}$. The media without the supplementation of HA were included as a control. The cells were continued culturing for another 4 days and the spheres were disrupted to form secondary spheres for an additional culturing of 2 days. Spheres reaching $100 \mu \mathrm{m}$ were considered as self-renewing and were used for the all the analysis.

\section{Mouse}

The nude mice (Balb/c-nu/nu, female, 4 weeks old) were obtained from Charles River, Japan. For all the transplantation studies, $1 \times 10^{6}$ cells were suspended in $200 \mu \mathrm{L}$ in complete media and were injected subcutaneously and intra dermally. Size and volume measurement were done every 3-4 days with the following formula $0.5 \times$ width $2 \times$ length, in which width is the smallest diameter and length is the longest diameter. After 5 weeks, tumors were extracted and separated in 4 equal parts that were used for the primary cell culture, histological analysis as described below. The animals were maintained in dedicated animal facility in Okayama University and were fed ad libitum. All the animal experiments conducted were carefully designed and executed as per the general guidelines stipulated by the ethics committee of Okayama University.

\section{RNA extraction and qRT PCR}

RNA extraction and quantitative PCR were performed as described elsewhere [10]. The primers used for the detect the cDNAs of interest are tabulated in Supplementary Table 1.

\section{Cell lysis and western blotting}

Total cell extracts were collected as follows: Cells were lysed in $300 \mu \mathrm{l}$ of lysis buffer (10 mM Tris-HCl, pH 7.5, 5 mM EDTA, $150 \mathrm{mM} \mathrm{NaCl}, 1 \%$ Triton-X 100, 10\%(v/v) glycerol containing protease inhibitor cocktail for $30 \mathrm{~min}$ at $4^{\circ} \mathrm{C}$. The lysate was then sonicated for 30 secs; 2 times and were centrifuged at $14,000 \mathrm{rpm}$ for $10 \mathrm{~min}$ at $4^{\circ} \mathrm{C}$ and the supernatant were collected for the subsequent analysis. Cell extracts isolated were then subsequently separated in polyacrylamide gels, transferred onto
PVDF membrane and incubated with specific antibodies as indicated. Immunoreactivity was revealed by chemiluminescense as per the manufacturer's instruction and measured using the Atto imaging system (Atto, Japan).

\section{Immunohistochemistry and histological analysis}

The tumor extracted from the animal were primarily fixed with paraformaldehyde for $24 \mathrm{hrs}$ followed by paraffin embedding procedure for histochemical analysis. The fixed tissue was then thoroughly washed and were permeated with increasing concentration of sucrose. Tissue blocks were made with OCT reagent (Sakura-Finetek, Netherlands) and were stored at $-80^{\circ} \mathrm{C}$ until processing. The samples were fixed with absolute methanol for $10 \mathrm{~min}$ followed by blocking of the endogenous peroxidase with $3 \% \mathrm{H}_{2} \mathrm{O}_{2}$ for $10 \mathrm{~min}$. Using the Ellite anti-rabbit $\mathrm{ABC}$ vectastain kit (Vector Laboratories, USA) anti-CD31 (1:100, \#ab28364, Abcam, UK), anti-CD133 (Novus Bio, USA) anti-LYVE-1 (Abcam, UK) antibodies were incubated for $2 \mathrm{hrs}$ at room temperature. Immunoreactivity were detected by using DAB following the manufacturer's recommendations. Images of the sections were processed with the FSX100 (Olympus, Japan) microscope for analysis and identification of the tumor type.

\section{Results and Discussion}

\section{HA mediates enrichment of CD44 expressing population}

High molecular weight $\mathrm{HA}$ stimulates the CD44 mediated clustering of breast cancer cells [15]. The CD44 binding to HA also has various outcomes based on the size of HA binding to CD44 such as proangiogenic and pro-inflammatory while nHA promoted anti-angiogenic and anti-inflammatory effects [16-18]. To assess the HA mediated enrichment of CD44, we cultured A172 and U251MG glioblastoma cells in both adherent and non-adherent conditions at a concentration of $100 \mu \mathrm{g} / \mathrm{ml}$ of HA. The cells in the adherent conditions without HA were bound to the plate while the spheroid formation could be seen with the non-adherent HA+ treated cells. Primary cultures (U251MG-P1) from the tumor-bearing mouse showed enrichment of more spheroid forming population when compared with the parental cell line (Figure 1A). The increment in the spheroid formation was further confirmed with culturing the A172, U251MG and U251MG-P1with (HA+) and without (HA-) the addition of HA in the culture medium (Figure 1B). The sphere formation was enhanced in U251MG-P1 resembling the stem cell morphology, while the parental cell line showed loosely formed aggregates with more differentiated cells.

To test the self-renewal in the tumor spheroids generated by the treatment, we dissociated the cells to single cells suspension with collagenase treatment and were cultured for 2 weeks with HA in adherent conditions (Figure 1C). The cells retained the spheroid forming ability which resembles that of the CSCs. The presence of HA-CD44 interaction in the cells promotes the aggregation and the CD44 over expressing population should be the reason for the reconstitution of the spheroid formation. The spheroid formation and the incidence time for the sphere formation were up regulated with the treatment of HA.

\section{CD44 expression is upregulated by $\mathrm{HA}$}

We next checked the expression of CD44 in A172, U251MG and U251MG-P1 cell lines by qPCR. The expression analysis was done between the cell lines treated with $\mathrm{HA}+$ and HA- and between the adherent culture and non-adherent culture. In U251MG and A172 cell lines the CD44 expression was up regulated 3 folds in non-adherent HA+ cells when compared with adherent HA-counterpart (Figure 2A and 2B). Similar results were obtained between U251MG and U251MG-P1 
Citation: Vaidyanath A, Mahmud HB, Khayrani AC, Oo AKK, Seno A, et al. (2017) Hyaluronic Acid Mediated Enrichment of CD44 Expressing Glioblastoma Stem Cells in U251MG Xenograft Mouse Model. J Stem Cell Res Ther 7: 384. doi: 10.4172/2157-7633.1000384

A

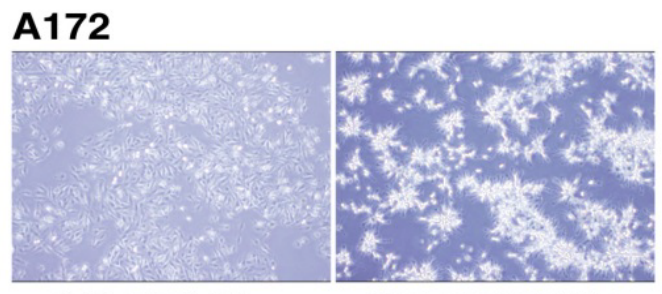

Adherent HA-

Non-coated HA+

\section{U251MG-P1}

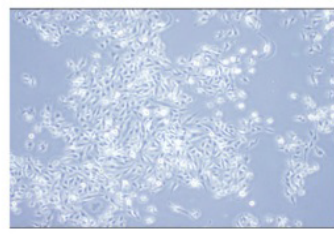

Adherent HA-

B

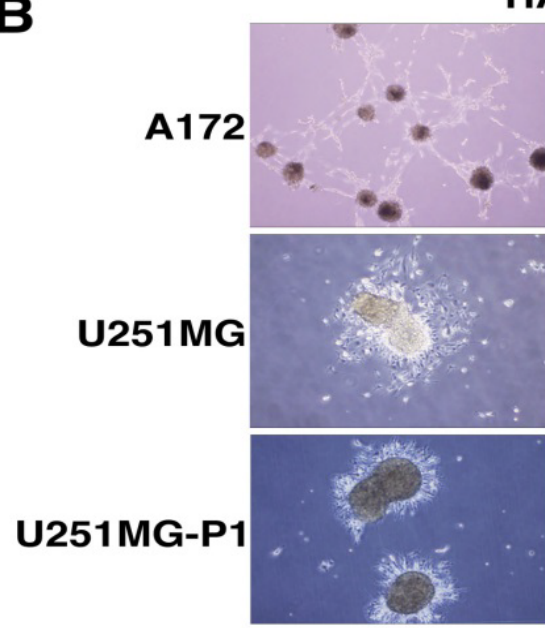

$4 X$

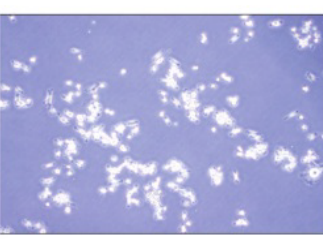

Non-coated HA-
U251MG

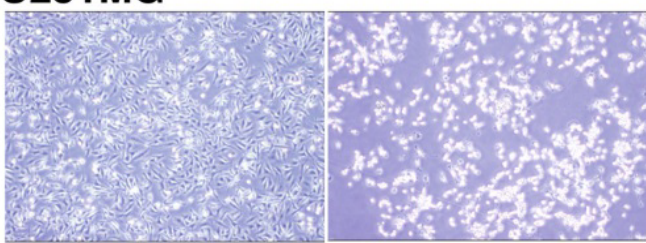

Adherent HA-

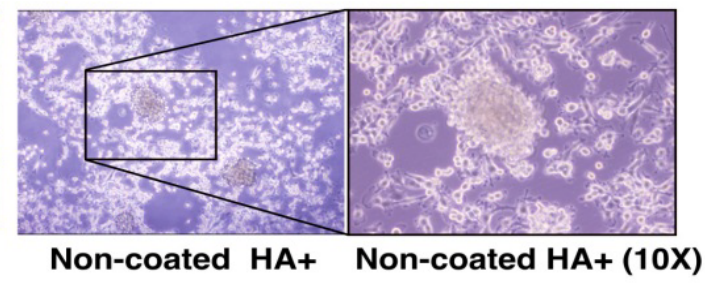

HA-

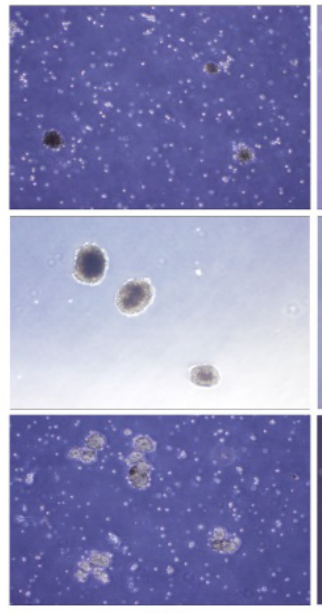

4X

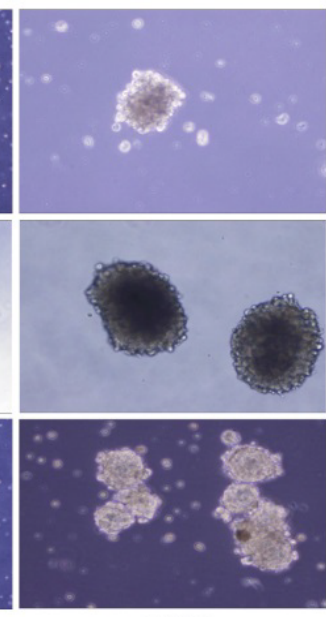

$10 X$

C

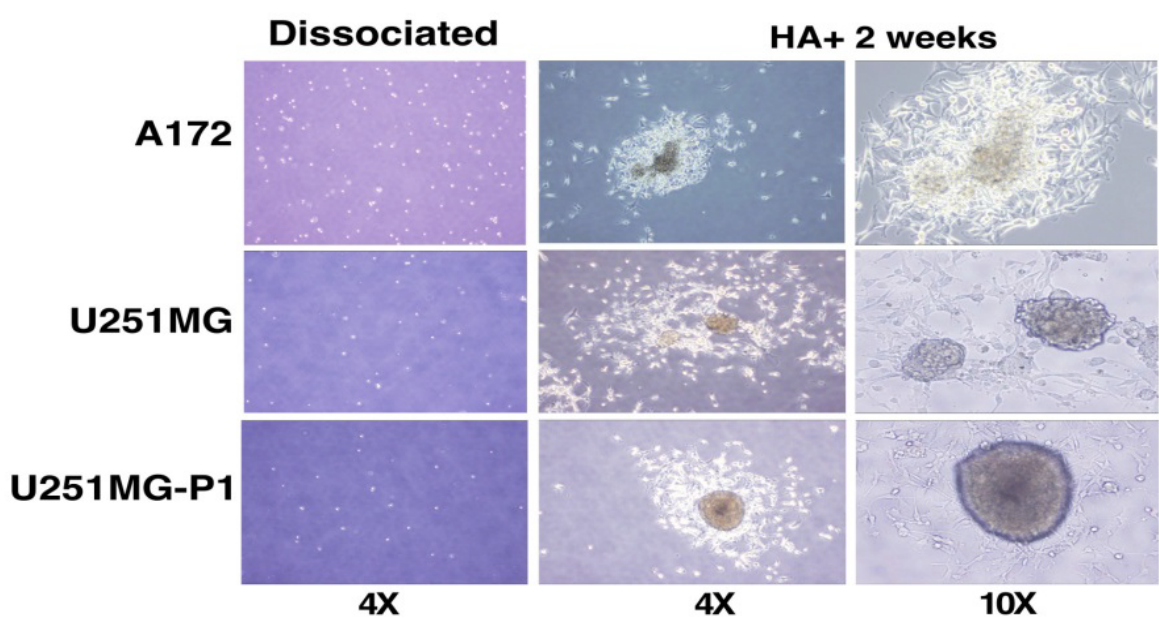

Figure 1: Induction of U251MG cells with HA. (A) Cell morphology of A172, U251MG and U251MG-P1 cells. Cells were cultured in culture dish with (100 $\mu$ g/ml $\mathrm{HA}$ ) and without HA in non-adherent conditions. Inset shows the magnified image of the spheroid formation in HA+ dish. (B). Spheroid formation of A172, U251MG, U251MG-P1 cells in the presence or absence of HA for 2 weeks. (C) Cell Morphology of A172, U251MG and primary culture of U251MG (U251MG-P1) after dissociation and culturing with HA for 2 weeks in adherent conditions. 
A

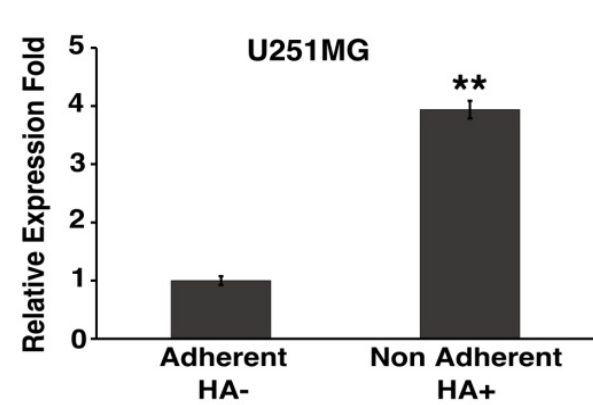

B

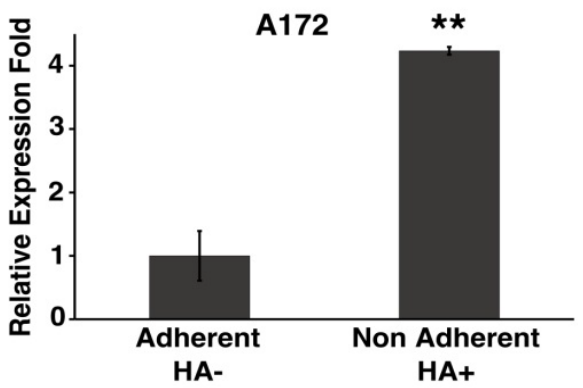

C

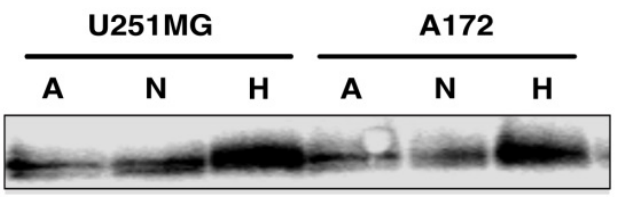

\section{CD44}

\section{$\beta-A C T I N$}

D

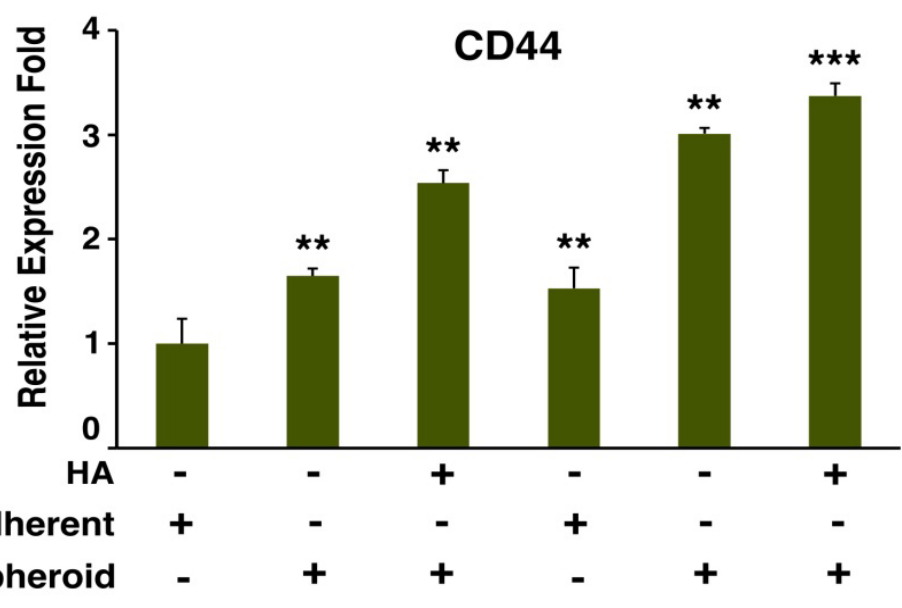

$\mathbf{E}$

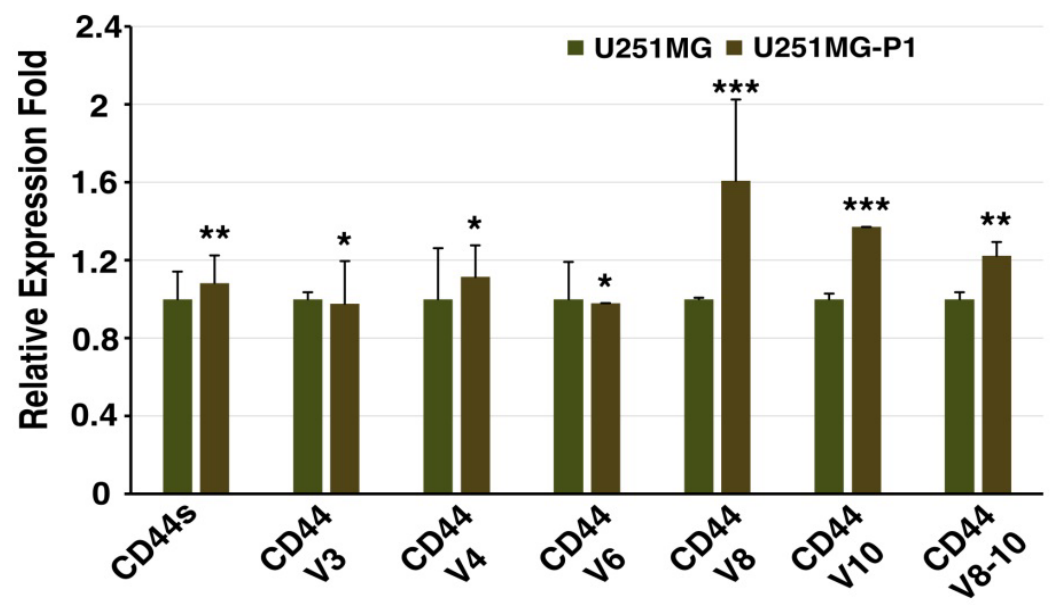

Figure 2: Up regulation of CD44 expression in U251MG cells. CD44 expression of adherent HA- and nonadherent HA+ treatment of U251MG (A) and A172 (B) by qPCR. Protein expression was further confirmed with the various treatment in A172 and U251MG cells A: Adherent, N: Non-adherent, $H$ : Non-adherent HA+ (C). U251MG and U251MG-P1 cells were cultured under adherent and nonadherent conditions with or without the supplementation of HA. qPCR was performed and relative expression fold of CD44 was calculated (E) Quantitative PCR analysis of the expression of CD44 isoforms in U251MG and U251MG-P1 cells in adherent conditions. The data depicted are generated from two independent experiments. $p$ values were calculated by Tukey HSD analysis $\left({ }^{\star *} p<0.01 ;{ }^{* \star \star} p<0.001 ; n=2\right)$. 
cells were the up regulation were found to be 2 -fold when compared with adherent HA- conditions. The gene expression was further checked in the protein expression level through immunoblots with the cells. The immunoblot results clearly show that the HA-treated cells express more CD44 when compared to non-treated samples (Figure 2C). We further compared the enrichment of the CD44 expression in the population of cells U251MG and U251MG-P1 in the adherent and spheroid conditions with and without the induction of HA. All our results from these experiments proved that the CD44 expression is considerably up regulated by the treatment with HA there by the enrichment of the CD44 expressing population (Figure 2D). We further analyzed the effect of CD44 isoform expression in U251MG and U251MG-P1 cells. We found the expression of stable, V4, V8, V10 and V8-10 to be up-regulated in U251MG-P1 (Figure 2E).

\section{HA induces the expression of pluripotent genes}

Aberrant activation of the pluripotent genes augments cancer initiation, progression, and chemotherapeutic resistance. These transcription factors are found to be over-expressed in several of the cancers and were used to identify cancer stem cell-like cells in glioblastoma [19]. HA-CD44 interaction was proved to activate Nanog which is principally [20] involved in the stem cell maintenance and self-renewal in the ES cells $[21,22]$. The activation of CD44 through the PKCe phosphorylates Nanog, with or without the association of Stat3 regulates the expression of other pluripotent, tumorigenic and multidrug resistance genes [21]. Nanog has shown to regulate the expression of Sox 2 , Oct $3 / 4$, and Rex1, the prominent pluripotent transcription factors during embryonic stem cell pluripotency. To assess the activation of embryonic pluripotency genes we performed the qPCR for the various treatment of the cells in the conditions depicted in Figure 3. The cells treated with HA augments the expression of pluripotency genes suggesting the possible acquiring of stem cell characteristics (Figure $3 \mathrm{~A}$ ). Since Sox 2 has been proved to be the principal pluripotency genes in the generation of CSC we analyzed the expression further with immunoblots (Figure 3B).

\section{Generation of glioblastoma mouse model}

Using the HA-induced CD44 up regulation we developed a glioblastoma mouse CSC model. We injected the spheres cultured in the presence of HA into the mouse subcutaneously. The tumor incidence time was faster in the treated spheres within 40 days when the adherent culture injected mouse showed no signs of tumor formation substantiating tumor-initiating population in the HA cultured spheroids. HA-treated spheroids generated tumors faster in the mouse by several folds when compared with the cultures of U251MG cells cultured without HA in adherent conditions. Thus, the CD44 induction with the non-adherent conditions is found to be the deciding factor for the reduced tumor latency in the mouse in question. We also assessed the tumor volume and the weight of the animal throughout the procedure. With the increment in the tumor volume, the weight of the animal remained within the acceptable range of 5\% difference between the groups, when compared to the control (Figure 4). To evaluate the extent of differentiation in these tumors we also analyzed the Glial Fibrillary Acidic Protein (GFAP), a marker used to distinguish astrocytes from glial cells which also is a progenitor marker for the Neural Stem Cells (NSCs). In our hands, the GFAP expression is reduced in the U251MG-P1 cells which might be due to the enrichment of the undifferentiated stem-like the population in the primary tumor from the mouse (Supplementary
Figure 1A). To further probe the stability of the GFAP expression in the tumor we subsequently retransplanted the tumor to the mice and were further confirmed with the western blot (Supplementary Figure 1A). Immunohistochemical analysis showed the extent of angiogenesis induction along with the presence of CD133+ cells intercalated throughout the tumor. We further probed for the expression of LYVE1 to check the lymphangiogenesis in the tumor and in our hands, the LYVE-1 expression was very much limited in the tumor (Supplementary Figure 1B). Histopathological and gene expression analysis have further confirmed the similar characteristic of the parental cancer cell line.

Similar studies have been conducted in breast and colon cancer previously $[23,24]$ along with combining the genetic model with xenograft transplantations [25]. Similar studies has shown that the $\mathrm{CD} 133+$ conditioned media in glioma enhances the angiogenesis in patient xenografts in mouse by elevated VEGF expression and endothelial differentiation thereby the presence of stem cell populations determines the key events in angiogenesis and vascular mimicry [26-28]. Similar model system was generated using miPS cells by our group where the angiogenesis/vascular mimicry were a cooperative interaction between the CSC and the microenvironment comprising of the differentiated populations, including the endothelial cells, and tumor stroma [29].

\section{Activation of NFKB signaling in U251MG-P1 cells}

NFKB complex activates MMP-9 and several other genes which are key response elements in the tumorigenesis and stem cell maintenance through tumor microenvironment mediated inflammation and ECM signaling [30] [31,32]. To test the activation of inflammatory mediators we checked the NFkB activation in the HA-treated spheroids. NFkB activation has been associated with the mesenchymal subtype of the glioma and is proved to provide radioresistance in cancer [33]. We found that the HA induces p50/105 activation in the U251MG-P1 cells consistent with the results got from the high-grade glioblastoma models. Further, we found that p65 is activated in both parental and U251MG-P1 cells. Based on these observations we then checked for the genes involved in the NFKB family by quantitative PCR and we found that the NFKB1, which is essential for the translocation of the p50/65 complex into the nucleus for the gene regulation, was suppressed, and REL-B was found to be expressing in the U251MG-P1 cells showing the mesenchymal nature of the primary tumor cells [34] (Supplementary Figure 2).

\section{Conclusion}

Xenograft models give more simultaneity and reproducibility with their high incidence of the tumor formation Also they allow easy tumor visualization for the drug development process and efficient penetrance of the drug interactions with the tumor [35,36]. Further the establishment of the model system will be advantageous for the replenishment of CSC, as in long turn in vitro culture system these cells undergo differentiation to normal cancer cells. Enrichment of CD44 expressing cells would be a better platform in the drug targeting experiments for CSCs as we previously successfully showed targeting HER2 breast cancer cells with our bionanocapsule [37] and liposome [38,39] with HER2-binding artificial ligands and such models will provide extensive knowledge in the therapeutic intervention in curbing the cancer growth and metastasis. The advantage of our model involves its cost-effective enrichment of the CD44 expressing glioblastoma cells 
A
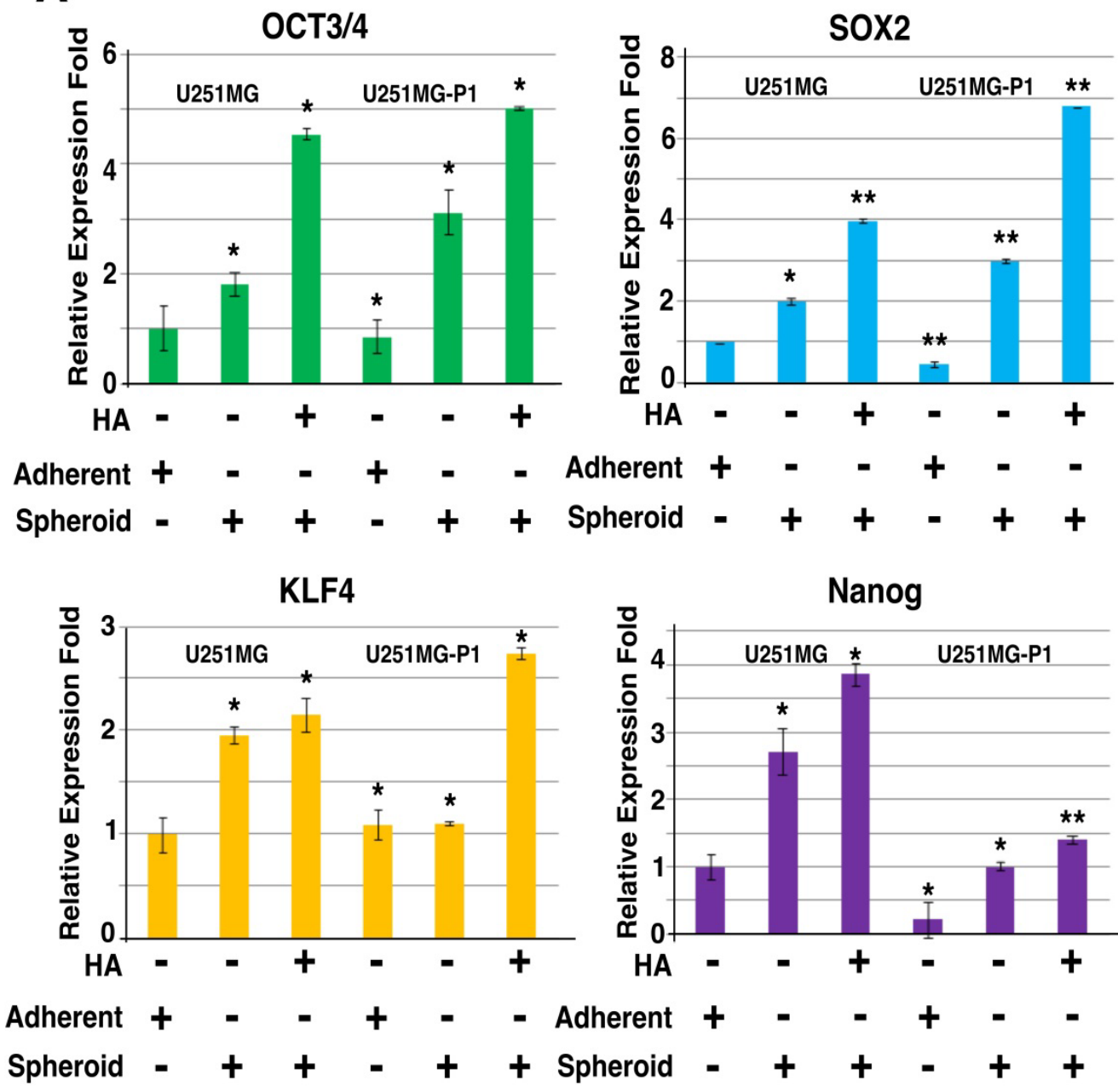

B
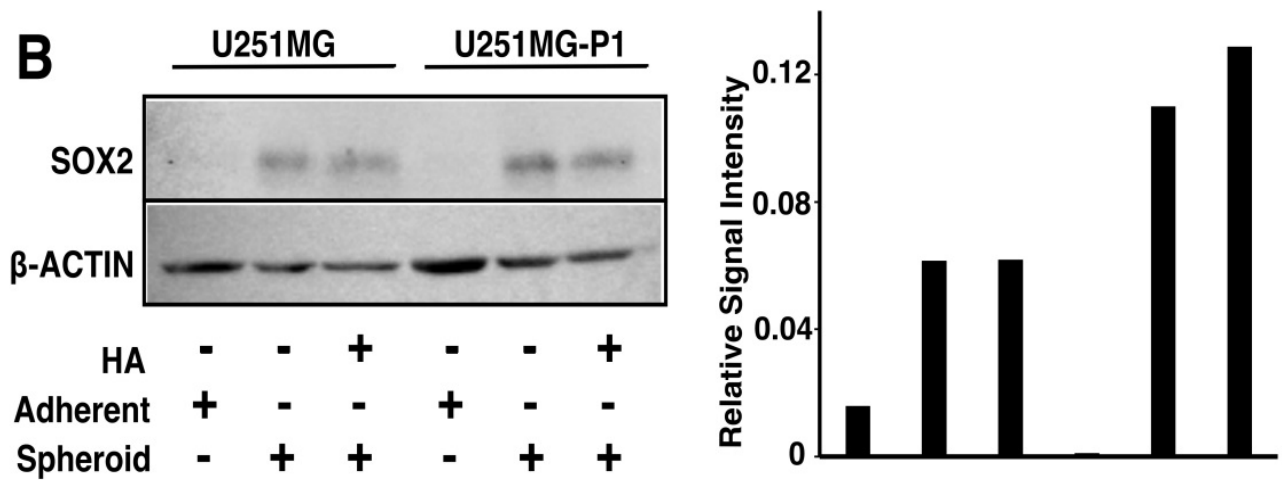

Figure 3: Expression of pluripotent genes in U251MG and U251M-P1 cells. (A) Cells were cultured with or without HA in adherent or nonadherent conditions. qPCR analysis was performed with the primers for Sox2, Oct3/4, KIf4 and Nanog. Error bars represents the mean SD of two independent experiments. Data are the mean of independent experiments and the $p$ values were calculated by Tukey HSD analysis $\left({ }^{*} p<0.05 ;{ }^{* *} p<0.01 ; n=3\right)(B)$ Cells cultured under the different conditions were lysed, separated on SDS-PAGE and were immunoblotted for Sox2 expression. The normalized relative band intensity was calculated from the blot and were plotted as a graph using Image $\mathrm{J}$. 
Citation: Vaidyanath A, Mahmud HB, Khayrani AC, Oo AKK, Seno A, et al. (2017) Hyaluronic Acid Mediated Enrichment of CD44 Expressing Glioblastoma Stem Cells in U251MG Xenograft Mouse Model. J Stem Cell Res Ther 7: 384. doi: 10.4172/2157-7633.1000384

A

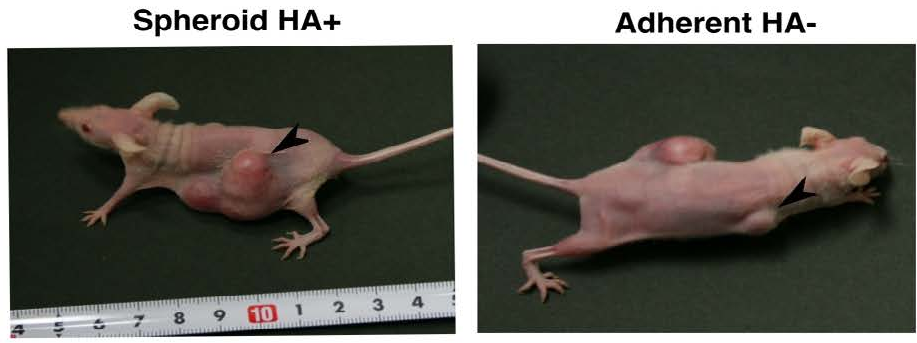

B

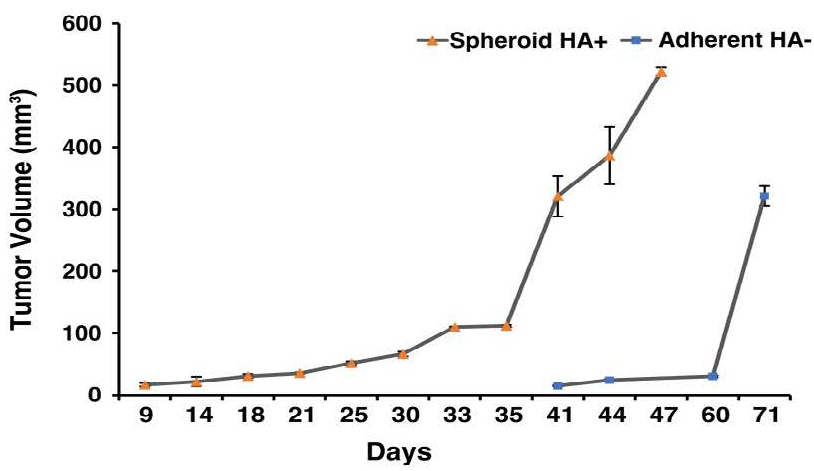

Figure 4: Characterization of mouse model of glioma. (A) Tumor formation in mouse with U251MG spheroids treated with HA and adherent culture of U251MG without the HA treatment. (B) Tumor incidence rate with spheroid/HA+ and adherent/HA- cultures of U251MG cells; $n=2$.

and high tumor incidence within a short time, enables us to study the effect of chemotherapeutic targeting. Further, the development of the mouse model will assist in the molecular targeting using anti-CD44 antibody-drug encapsulated nanoparticles for glioma CSCs.

\section{Acknowledgements}

This research was supported by the Grant-in-Aid for Scientific Research (A) No. 25242045 (MS); the Grant-in-Aid for Challenging Exploratory Research No. 26640079 (MS); the Japan Science and Technology Agency, Matching Planner Program-Tansaku Shiken-Grant (TK) and Wesco Scientific Promotion Foundation (TK). Authors appreciate Dr. Akifumi Mizutani for his guidance and support.

\section{Conflict of Interest}

All the authors declare no competing financial interest

\section{References}

1. Cancer Genome Atlas Research Network (2008) Comprehensive genomic characterization defines human glioblastoma genes and core pathways. Nature 7216: 1061-1068. [PubMed]

2. Louis DN, Ohgaki H, Wiestler OD, Cavenee WK, Burger PC, et al. (2007) The 2007 WHO classification of tumours of the central nervous system. Acta Neuropathol 2: 97-109. [PubMed]

3. Wiranowska M, Rojiani MV (2011) Extracellular Matrix Microenvironment in Glioma Progression. INTECH Open Access Publisher Rijeka, Croatia.

4. Zhan C, Lu W (2012) The blood-brain/tumor barriers: challenges and chances for malignant gliomas targeted drug delivery. Curr Pharm Biotechnol 12: 23802387. [PubMed]

5. Plaks V, Kong N, Werb Z (2015) The cancer stem cell niche: how essential is the niche in regulating stemness of tumor cells? Cell Stem Cell 3: 225-238. [PubMed]

6. Nguyen LV, Vanner R, Dirks P, Eaves CJ (2012) Cancer stem cells: an evolving concept. Nat Rev Cancer 2: 133-143. [PubMed]

7. Egeblad M, Nakasone ES, Werb Z (2010) Tumors as organs: complex tissues that interface with the entire organism. Dev Cell 6: 884-901. [PubMed]
8. Tammi RH, Kultti A, Kosma VM, Pirinen R, Auvinen P, et al. (2008) Hyaluronan in human tumors: pathobiological and prognostic messages from cell-associated and stromal hyaluronan. Semin Cancer Biol 4: 288-295. [PubMed]

9. Auvinen P, Rilla K, Tumelius R, Tammi M, Sironen R, et al. (2013) Hyaluronan synthases (HAS1-3) in stromal and malignant cells correlate with breast cancer grade and predict patient survival. Breast Cancer Res Treat 2: 277-286. [PubMed]

10. Sugii Y, Kasai T, Ikeda M, Vaidyanath A, Kumon K, et al. (2016) A Unique Procedure to Identify Cell Surface Markers Through a Spherical Self-Organizing Map Applied to DNA Microarray Analysis. Biomark Cancer: 17-23. [PubMed]

11. Chanmee T, Ontong P, Mochizuki N, Kongtawelert P, Konno K, et al. (2014) Excessive hyaluronan production promotes acquisition of cancer stem cell signatures through the coordinated regulation of Twist and the transforming growth factor $\beta$ (TGF- $\beta$ )-Snail signaling axis. J Biol Chem 38: 26038-26056. [PubMed]

12. Schatton T, Frank NY, Frank MH (2009) Identification and targeting of cancer stem cells. Bioessays 10: 1038-1049. [PubMed]

13. Ponta H, Sherman L, Herrlich PA (2003) CD44: from adhesion molecules to signalling regulators. Nat Rev Mol Cell Biol 1: 33-45. [PubMed]

14. Pietras A, Katz AM, Ekström EJ, Wee B, Halliday JJ, et al. (2014) OsteopontinCD44 signaling in the glioma perivascular niche enhances cancer stem cell phenotypes and promotes aggressive tumor growth. Cell Stem Cell 3: 357-369. [PubMed]

15. Yang C, Cao M, Liu H, He Y, Xu J, et al. (2012) The high and low molecular weight forms of hyaluronan have distinct effects on CD44 clustering. J Biol Chem 51: 43094-43107. [PubMed]

16. Zöller M (2011) CD44: can a cancer-initiating cell profit from an abundantly expressed molecule? Nat Rev Cancer 4: 254-267. [PubMed]

17. Gao F, Yang CX, Mo W, Liu YW, He YQ (2008) Hyaluronan oligosaccharides are potential stimulators to angiogenesis via RHAMM mediated signal pathway in wound healing. Clin Invest Med 3: E106-116. [PubMed]

18. Wang YZ, Cao ML, Liu YW, He YQ, Yang CX, et al. (2011) CD44 mediates oligosaccharides of hyaluronan-induced proliferation, tube formation and signal transduction in endothelial cells. Exp Biol Med 1: 84-90. [PubMed] 
Citation: Vaidyanath A, Mahmud HB, Khayrani AC, Oo AKK, Seno A, et al. (2017) Hyaluronic Acid Mediated Enrichment of CD44 Expressing Glioblastoma Stem Cells in U251MG Xenograft Mouse Model. J Stem Cell Res Ther 7: 384. doi: 10.4172/2157-7633.1000384

Page 8 of 8

19. Olmez I, Shen W, McDonald H, Ozpolat B (2015) Dedifferentiation of patientderived glioblastoma multiforme cell lines results in a cancer stem cell-like state with mitogen-independent growth. J Cell Mol Med 6: 1262-1272. [PubMed]

20. Bourguignon LYW, Spevak CC, Wong G, Xia W, Gilad E (2009) HyaluronanCD44 Interaction with Protein Kinase C Promotes Oncogenic Signaling by the Stem Cell Marker Nanog and the Production of MicroRNA-21, Leading to Down-regulation of the Tumor Suppressor Protein PDCD4, Anti-apoptosis, and Chemotherapy Resistance in Breast Tumor Cells. J Biol Chem 39: 2653326546. [PubMed]

21. Bourguignon LYW, Peyrollier K, Xia W, Gilad E (2008) Hyaluronan-CD44 interaction activates stem cell marker Nanog, Stat-3-mediated MDR1 gene expression, and ankyrin-regulated multidrug efflux in breast and ovarian tumor cells. J Biol Chem 25: 17635-17651. [PubMed]

22. Chambers I, Colby D, Robertson M, Nichols J, Lee S, et al. (2003) Functional expression cloning of Nanog, a pluripotency sustaining factor in embryonic stem cells. Cell 5: 643-655. [PubMed]

23. Singh SK, Clarke ID, Hide T, Dirks PB (2004) Cancer stem cells in nervous system tumors. Oncogene 43: 7267-7273. [PubMed]

24. Singh SK, Clarke ID, Terasaki M, Bonn VE, Hawkins C, et al. (2003) Identification of a cancer stem cell in human brain tumors. Cancer Res 18 : 5821-5828. [PubMed]

25. Harris MA, Yang H, Low BE, Mukherjee J, Mukherje J, et al. (2008) Cancer stem cells are enriched in the side population cells in a mouse model of glioma. Cancer Res 24: 10051-10059. [PubMed]

26. Bao S, Wu Q, Sathornsumetee S, Hao Y, Li Z, Hjelmeland AB, et al. (2006) Stem Cell-like Glioma Cells Promote Tumor Angiogenesis through Vascular Endothelial Growth Factor. Cancer Res 16: 7843-7848. [PubMed]

27. Chiao MT, Yang YC, Cheng WY, Shen CC, Ko JL (2011) CD133+ glioblastoma stem-like cells induce vascular mimicry in vivo. Curr Neurovasc Res 3: 210 219. [PubMed]

28. Ricci-Vitiani L, Pallini R, Biffoni M, Todaro M, Invernici G, et al. (2010) Tumour vascularization via endothelial differentiation of glioblastoma stem-like cells. Nature 7325: 824-828. [PubMed]

29. Prieto-Vila M, Yan T, Calle AS, Nair N, Hurley J et al. (2016) iPSC-derived cancer stem cells provide a model of tumor vasculature. Am J Cancer Res 9 : 1906-1921. [PubMed]

30. Bhat KPL, Balasubramaniyan V, Vaillant B, Ezhilarasan R, Hummelink K, et al. (2013) Mesenchymal differentiation mediated by NF-KB promotes radiation resistance in glioblastoma. Cancer Cell 3: 331-346. [PubMed]

31. Lee DW, Ramakrishnan D, Valenta J, Perney IF, Bayless KJ et al. (2013) The NF-kB RelB protein is an oncogenic driver of mesenchymal glioma. PLoS One 2: e57489. [PubMed]

32. Sen E (2011) Targeting inflammation-induced transcription factor activation: an open frontier for glioma therapy. Drug Discov Today 23-24: 1044-1051. [PubMed]

33. Nogueira L, Ruiz-Ontañon $P$, Vazquez-Barquero A, Lafarga $M$, Berciano MT et al. (2011) Blockade of the NFKB pathway drives differentiating glioblastomainitiating cells into senescence both in vitro and in vivo. Oncogene 32: 35373548. [PubMed]

34. Kim MS, Park MJ, Kim SJ, Lee CH, Yoo H, et al. (2005) Emodin suppresses hyaluronic acid-induced MMP-9 secretion and invasion of glioma cells. Int $\mathrm{J}$ Oncol 3: 839-846. [PubMed]

35. Fomchenko El, Holland EC (2006) Mouse models of brain tumors and their applications in preclinical trials. Clin Cancer Res 18: 5288-5297. [PubMed]

36. Chen J, McKay RM, Parada LF (2012) Malignant glioma: lessons from genomics, mouse models, and stem cells. Cell 1: 36-47. [PubMed]

37. Vaidyanath A, Hashizume T, Nagaoka T, Takeyasu N, Satoh H, et al. (2011) Enhanced internalization of ErbB2 in SK-BR-3 cells with multivalent forms of an artificial ligand. J Cell Mol Med 11: 2525-2538. [PubMed]

38. Shigehiro T, Kasai T, Murakami M, Sekhar SC, Tominaga Y, et al. (2014) Efficient drug delivery of Paclitaxel glycoside: a novel solubility gradient encapsulation into liposomes coupled with immunoliposomes preparation. PLoS One 9 : e107976. [PubMed]

39. Shigehiro T, Zhai W, Vaidyanath A, Masuda J, Mizutani A, et al. (2016) Evaluation of glycosylated docetaxel-encapsulated liposomes prepared by remote loading under solubility gradient. J Microencapsul 2: 172-182. [PubMed] 